\title{
Microarray profiling shows distinct differences between primary tumors and commonly used preclinical models in hepatocellular carcinoma
}

Weining Wang ${ }^{1}$, N. Gopalakrishna lyer ${ }^{1,2}$, Hsien Ts'ung Tay ${ }^{3}$, Yonghui Wu' ${ }^{1}$ Tony K. H. Lim, Lin Zheng ${ }^{5}$, In Chin Song ${ }^{5}$, Chee Keong Kwoh ${ }^{6}$, Hung Huynh${ }^{7}$, Patrick O. B. Tan ${ }^{8}$ and Pierce K. H. Chow ${ }^{2,9,10^{*}}$

\begin{abstract}
Background: Despite advances in therapeutics, outcomes for hepatocellular carcinoma (HCC) remain poor and there is an urgent need for efficacious systemic therapy. Unfortunately, drugs that are successful in preclinical studies often fail in the clinical setting, and we hypothesize that this is due to functional differences between primary tumors and commonly used preclinical models. In this study, we attempt to answer this question by comparing tumor morphology and gene expression profiles between primary tumors, xenografts and HCC cell lines.

Methods: Hep G2 cell lines and tumor cells from patient tumor explants were subcutaneously (ectopically) injected into the flank and orthotopically into liver parenchyma of Mus Musculus SCID mice. The mice were euthanized after two weeks. RNA was extracted from the tumors, and gene expression profiling was performed using the Gene Chip Human Genome U133 Plus 2.0. Principal component analyses (PCA) and construction of dendrograms were conducted using Partek genomics suite.

Results: PCA showed that the commonly used HepG2 cell line model and its xenograft counterparts were vastly different from all fresh primary tumors. Expression profiles of primary tumors were also significantly divergent from their counterpart patient-derived xenograft (PDX) models, regardless of the site of implantation. Xenografts from the same primary tumors were more likely to cluster together regardless of site of implantation, although heat maps showed distinct differences in gene expression profiles between orthotopic and ectopic models.

Conclusions: The data presented here challenges the utility of routinely used preclinical models. Models using HepG2 were vastly different from primary tumors and PDXs, suggesting that this is not clinically representative. Surprisingly, site of implantation (orthotopic versus ectopic) resulted in limited impact on gene expression profiles, and in both scenarios xenografts differed significantly from the original primary tumors, challenging the long-held notion that orthotopic PDX model is the gold standard preclinical model for HCC.
\end{abstract}

Keywords: Hepatocellular carcinoma, Ectopic, Orthotopic, Xenograft, HepG2 cell line

\footnotetext{
* Correspondence: pierce.chow@duke-nus.edu.sg

2Department of Surgical Oncology, National Cancer Centre Singapore,

Singapore 169610, Singapore

${ }^{9}$ Program in Translational and Clinical Liver Research, National Cancer Centre

Singapore, Singapore 169610, Singapore

Full list of author information is available at the end of the article
} 


\section{Background}

HCC is the third most common cause of cancer-related death [1-3], and the sixth most common cancer worldwide owing to increases in the prevalence of hepatitis $\mathrm{B}$ virus (HBV) and hepatitis $\mathrm{C}$ virus (HCV) [4-10]. The incidence is alarmingly high in the developing world and is rising steadily across the developed world [11, 12]. Three quarters of all HCC occurs in Asian countries due to high local prevalence of chronic HBV infection [13-15]. High incidence regions include sub-Saharan Africa, East Asia and Southeast Asia (Singapore, China, Hong Kong, Taiwan, Korea and Japan) [16-18]. In general, HCC poses a great health threat in the Asia Pacific region $[19,20]$. Surgery provides the best cure for HCC, without which demise usually occurs within 6 to 9 months [21]. Even after liver resection, overall prognosis is poor [22-24]. 1-year survival rates after surgical resection are $80-90 \%$, falling to $41-74 \%$ at 5 years $[12,25,26]$. Unfortunately less than $20 \%$ of patients are surgical candidates because of advanced disease stage at presentation. Even with surgery, up to $80 \%$ of patients develop recurrence within five years of resection [24, 27-29].

Advanced HCC is refractory to conventional chemotherapies and the current standard-of-care sorafenib confers a human survival advantage of only 2.8 months despite tumor regression and suppression of metastasis in mice [30, 31]. Hence, there is an urgent need for an efficacious systemic therapy in both the palliative and adjuvant settings. Advances in understanding of the pathophysiological and molecular basis of HCC have been unmatched by major pharmacological success. Encouraging preclinical animal results all too often fail to translate into human success, and only $45 \%$ of clinical agents showing xenograft responses exhibit clinical activity [32].

Unfortunately, encouraging results from preclinical trials do not often translate into similar successes in patients. We postulate that this disparity could be due to the use of transformed immortalized commercial cell line xenografts and the ectopic implantation of animal models. In contrast, commercial cell lines have a strong track record in cancer research. They are easy to use, readily available and produce reproducible results. However, not all cancers can be immortalized. Those that do accumulate mutations with increasing passages as they adapt to the artificial environment they are cultivated in. Consequently, they often differ genetically and phenotypically from their originating tumors [33]. There is also the well-documented risk of cross-contamination of cell cultures from cells of different origins. Similarly, the subcutaneous implantation in murine models is also favoured in preclinical studies because it is easy to establish and manage and lends itself readily to quantization of tumor burden [34, 35]. However, in vivo anti-tumor activity in preclinical animal models does not correlate closely with therapeutic response in human cancers of the same histology [32]. Many authors have also highlighted the importance of tumor microenvironment on the biological behaviour of tumor cells [36]. Despite being more time-consuming, expensive and technically expensive, it has been suggested that orthotopic transplantation of tumor cells into the anatomical site where the cancer commonly arises will give a model which mimics the biological behaviour of tumor cells more closely. This is especially important as the interactions between host environment and tumor graft determine tumor cell expression profiles, levels of growth factors and nutrients, angiogenesis and metastasis [37].

In this study, we investigate and compare the effects of two factors (cell lines vs. patient explants; ectopic vs. orthotopic) on gene expression profiles of HCC tumors. We hypothesized that differences would be observed in gene expression between ectopically and orthotopically transplanted tumors, and also between fresh patient-explanted tumors and established commercial cell lines. The similarities or differences between the sites and tumor types could potentially direct areas for future study.

\section{Methods}

\section{Establishment of murine models}

This study received ethics board approval from the SingHealth Centralized Institutional Review Board, SingHealth Institutional Animal Care and Use Committee (IACUC) and SingHealth Institutional Biosafety Committee. All mice were maintained according to the Guide for the Care and Use of Laboratory Animals published by the National Institutes of Health, USA.

Hep G2 is an established cell line often used in research pertaining to $\mathrm{HCC}$ and was selected for comparison against patient tumor explants in our study [38]. The immortalized cell lines were provided by the National University of Singapore (NUS) Biochemistry Laboratory. These had been previously passaged through several generations after purchase from ATCC. Tumor tissue was obtained intraoperatively during liver resection from three patients with prior written informed consent from Department of General Surgery, Singapore General Hospital. All three patients had hepatocellular carcinoma confirmed by histology. The explants were named explant 261004, 21318 and 01-0207. Sections weighing approximately $300 \mathrm{mg}$ were minced into $1-2 \mathrm{~mm}^{3}$ fragments using surgical blades, filtered through an 18 Gauge needle and washed 3 times with RPMI1640 before suspension in $0.1 \mathrm{ml}$ of RPMI medium. These cells were then passaged through 5 generations before ectopic or orthotopic implantation into 9 pairs of mice. 
Mus Musculus SCID mice were purchased from Animal Resources Centre, Australia. They were maintained for 8 to 10 weeks before experiments in facilities approved by IACUC and the approving ethical committee was SingHealth IACUC. No animal research was conducted outside of the country of residence.

The mice were divided into four groups - ectopic models with Hep G2 cells or cells derived from tumor explants and orthotropic models with Hep G2 cells or cells derived from tumor explants. In order to establish ectopic models, cells were injected subcutaneously into the flank regions of 4 pairs of mice. For orthotopic models, 8 pairs of mice were anaesthetized pre-operatively using intraperitoneal injection of $50 \mathrm{mg} / \mathrm{kg} / 5 \mathrm{mg} / \mathrm{kg}$ of ketamine/diazepam solution followed by intramuscular injection of $5 \mathrm{mg} / \mathrm{kg}$ of baytril. After anesthetization, the left lobe of liver was exposed through midline abdominal incision and cells were injected directly into liver parenchyma of 4 pairs of mice for each orthotopic model. At any given time during the research study, animals suffering severe or chronic pain or unrelievable distress were painlessly euthanized.

In subcutaneous models, tumor size was measured using callipers. On the other hand, the tumor size of the mice in orthotopic models was monitored and imaged using an R4 microPET scanner (Concordes

\section{a}

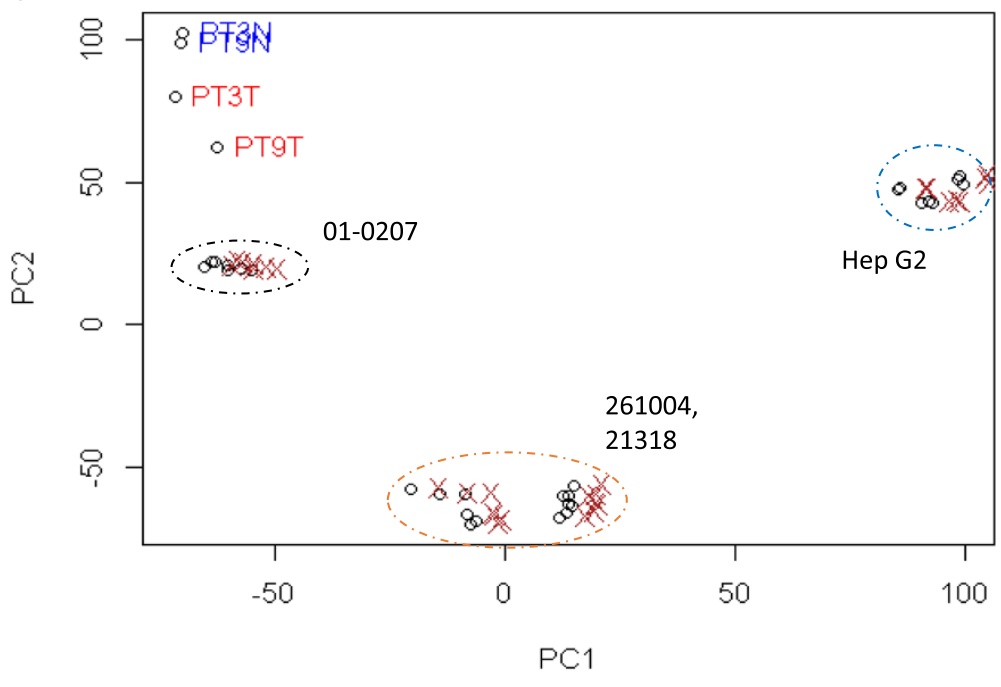

b

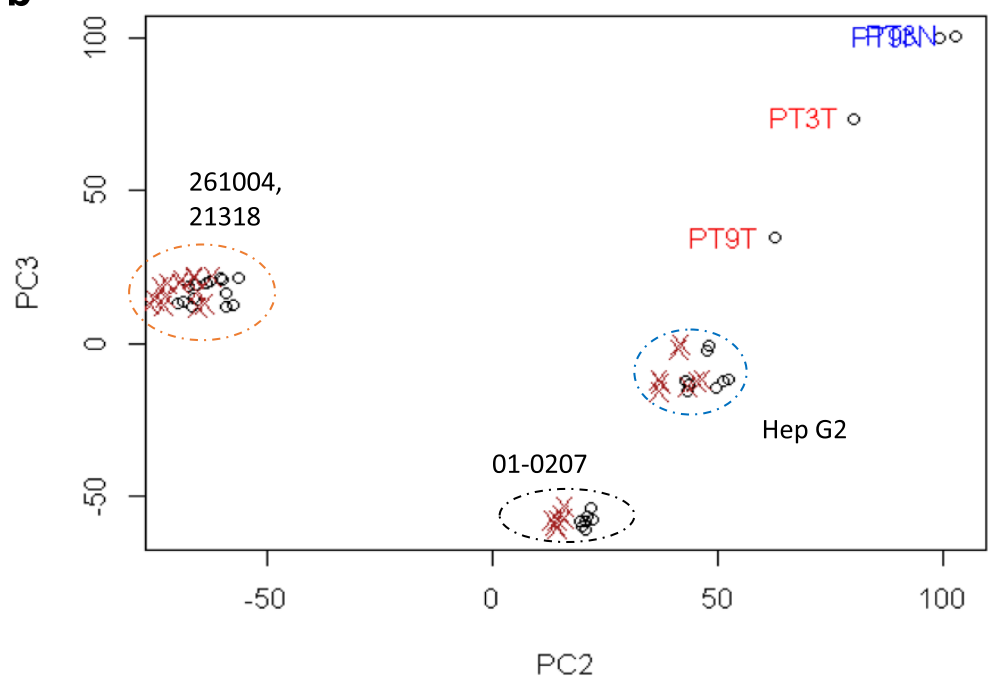

Fig. 1 PCA analysis of hierachial clustering of originating tumors, cell line and xenograft samples. a PC2 versus PC1, b PC3 versus PC2. The originating tumors are represented by spots annotated PTXX. Xenograft and cell lines are segregated from originating tumors. When compared within the murine models, Hep G2 and xenografts from the same primary tumors cluster together regardless of site of implantation 
Microsystems Inc., Knoxville, Tenn., USA). Prior to scanning, the mouse was anaesthetized using $2 \%$ isoflurane. $150-200 \mathrm{uCi}$ of 18F-fluorodeoxyglucose ([18F]-FDG) was injected via the tail vein and conscious uptake was allowed for an hour prior to scanning. The mouse was placed in prone position and static scanning was done for $10 \mathrm{~min}$. Analysis and quantitation of the microPET images were done using Asipro software.

At 2 weeks post-inoculation, tumor size had increased to approximately $1-2 \mathrm{~cm}$ in both models. The mice were then euthanized and the tumors were harvested.
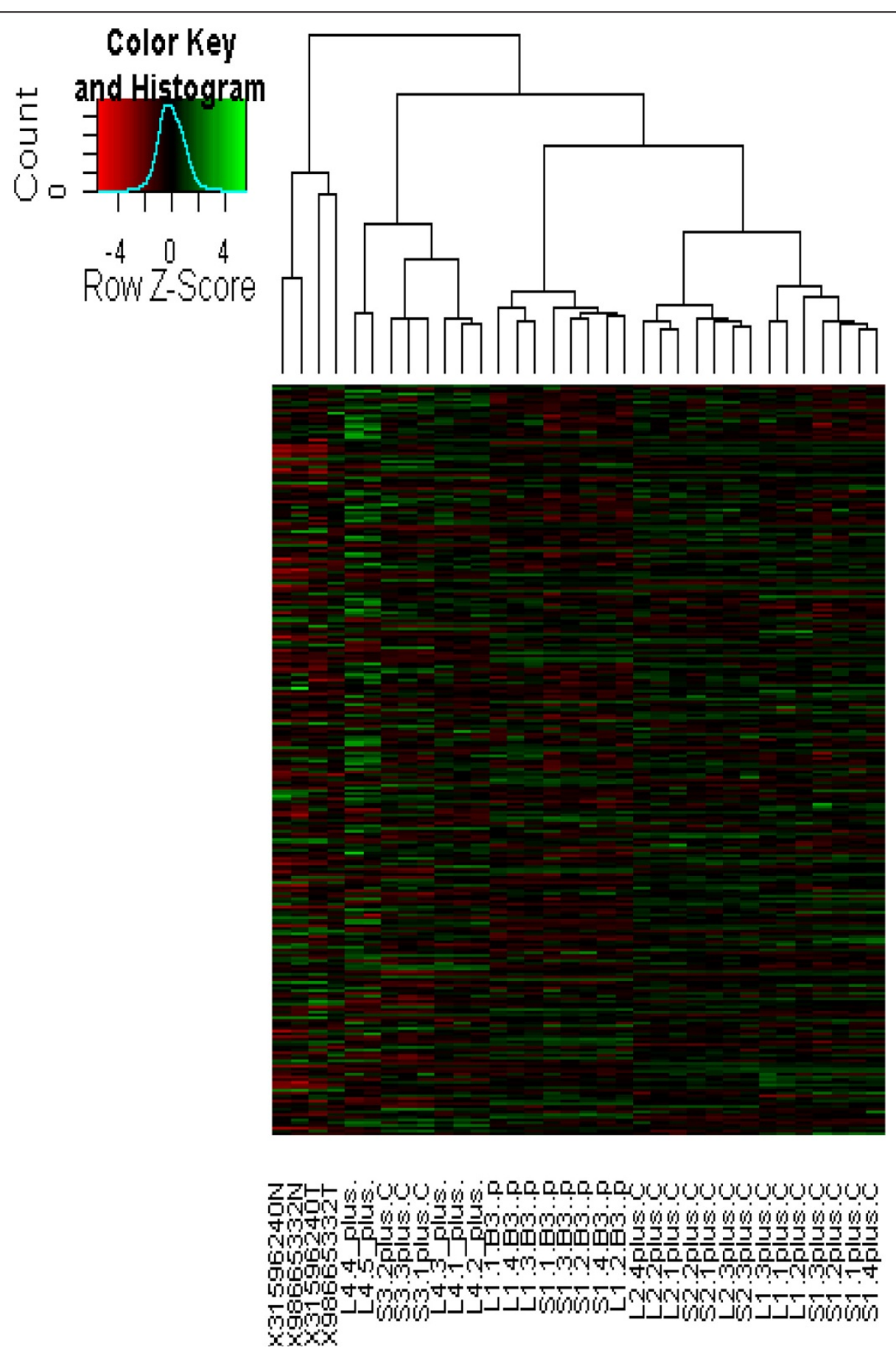

Fig. 2 Dendrogram showing clustering of originating tumors, cell line and xenograft samples. There was a distinct separation between originating tumors and samples from murine models but xenografts derived from the same originating tumor clustered together regardless of implantation site. The Hep G2 cell line samples were distinct from patient-derived xenografts 


\section{RNA extraction, assessment of nucleic acid purity}

20-30 g of fresh tumor tissue was harvested from each mouse for RNA extraction. RNA was also extracted from primary tumours. RNA samples were extracted and purified from tumors using methods described in the RNeasy Mini-Handbook [39]. To negate the random expression changes that may be specific to a xenograft, tissue samples from randomly paired mice within each subgroup were pooled together. RNA concentration and nucleotide purity were confirmed by measuring absorbance at 260 and $280 \mathrm{~nm}$ using the SHIMADZU UV1700 Pharma Spec spectrophotometer. Samples with $\mathrm{QC}<1.8$ were excluded from the analysis.

\section{Microarray analysis}

We used an Affymetrix GeneChip platform with single channel technology. Extracted RNA was labelled with streptavidin-phycoerythrin conjugate (SAPE) and hybridized to Affymetrix Gene Chip Human Genome U133 Plus 2.0 single arrays. These arrays were scanned using GeneChip Scanner 3000 and images are produced and analysed to give an intensity level for each probe. The intensity level corresponds to the level of hybridisation that occurs for each probe. The raw CEL files were then background corrected and normalized using the Robust Multichip Array (RMA) algorithm [39, 40]. PCA was performed and one way ANOVA was used to partition and identify the major sources of variation. Major nonbiological effects (batch effects) influencing gene expression values were tested and corrected for using "Partek
Genomics Suite" software. Unsupervised hierarchical clustering was then performed using average linkage and Euclidean distance between intensity readings of all probes as the distance metric. A dendrogram was constructed to illustrate the data.

To compare the differences in gene expressions between orthotopic and ectopic models within each explant/cell line, one-way ANOVA was performed on all gene probes. A false discovery rate (FDR) of less than 0.1 was considered significant. Heat maps were also generated from the RNA microarray data.

\section{Results}

Both the PCA map (Fig. 1) and the dendrogram (Fig. 2) showed the fresh, primary tumors segregating into distinctly separate clusters from all other models, suggesting that primary tumors were genetically distinct from both the immortalised cell line and xenograft models, whether orthotopically or ectopically implanted. When comparing within the murine models, there was a genetic disparity between the HepG2 cell line model and the explant models.

Additionally, the dendrogram showed that the samples of all three patient explant models clustered according to their originating explant, suggesting that tumors were most closely related to other tumors derived from the same originating tumor rather than the site of implantation or the respective primary tumors themselves. However, when comparing within the same tumor explant, there was no difference between orthotopically

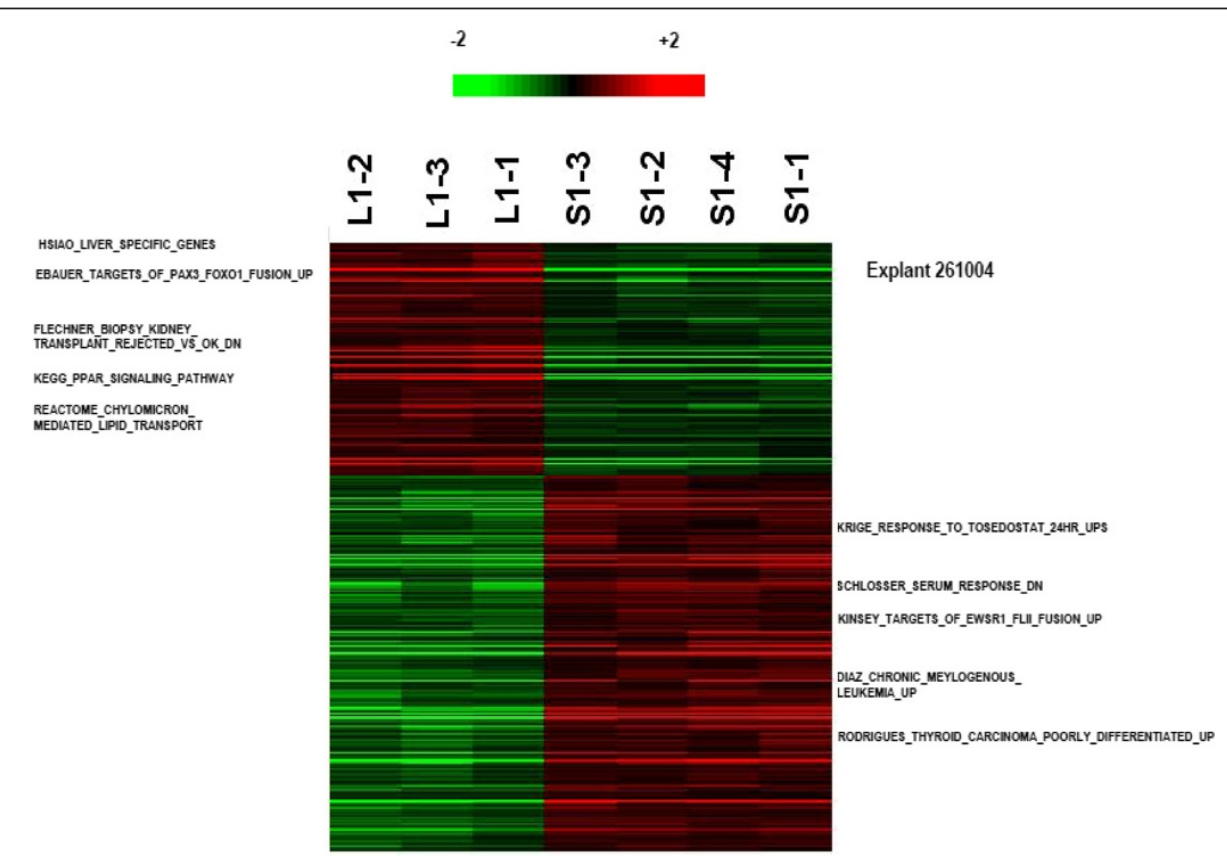

Fig. 3 Heat map comparing ectopic and orthotopic models of explant 261004. 396 probes and 56 probes were found to be significantly different between orthotopic and ectopic models of explant 261004 

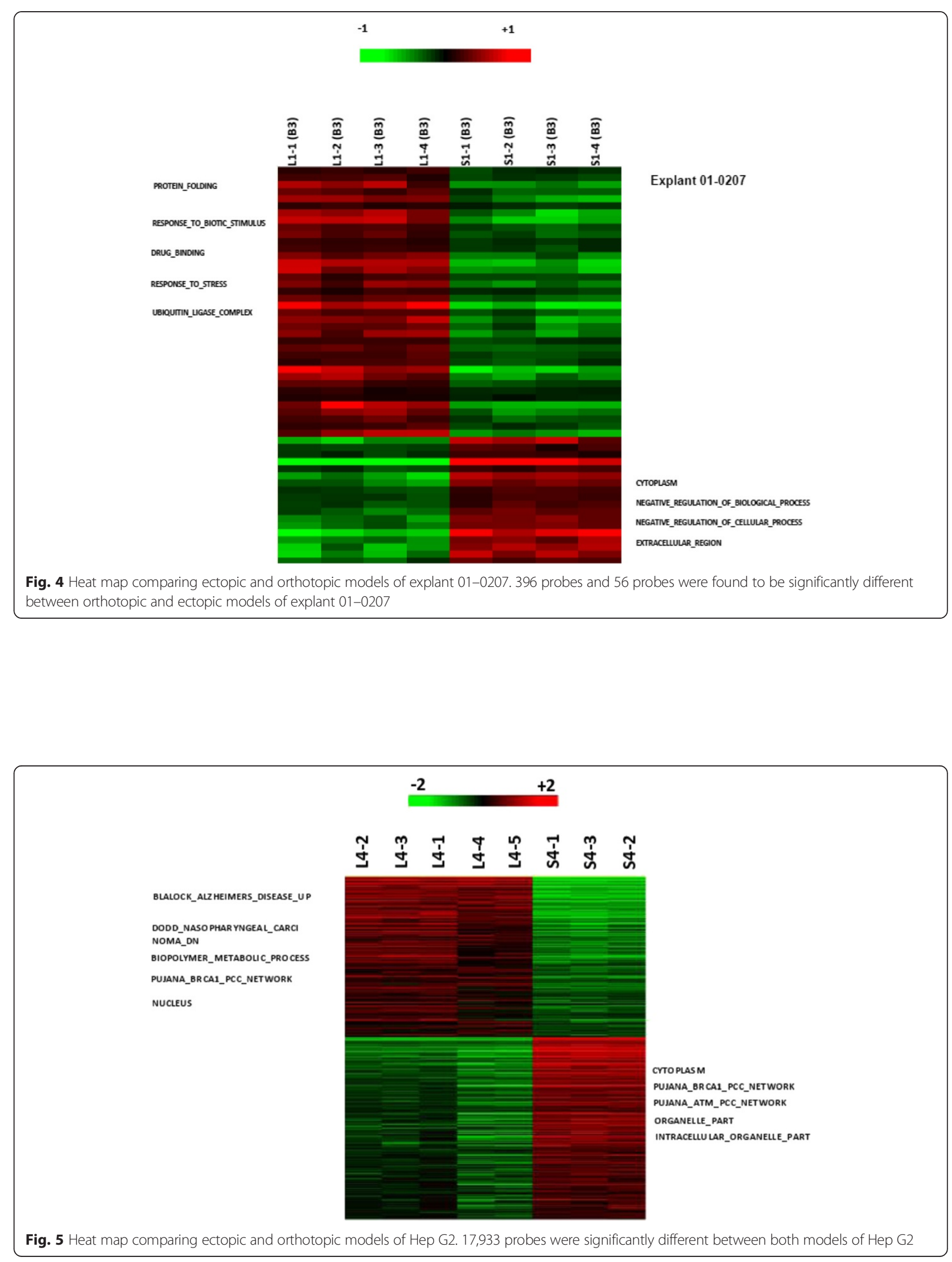
and ectopically implanted tumors. This corroborates with what is seen on the PCA map.

One-way ANOVA was performed on all 54,675 gene probes to compare the differences in gene expressions between the orthotopic and ectopic models within each explant/cell line. These results are demonstrated in the heat maps shown (Figs. 3, 4, and 5; Additional file 1). No probes were significantly different between orthotopic and ectopic samples of explant 21318. 396 probes and 56 probes were found to be significantly different for explants 261004 and 01-0207 respectively (Figs. 3 and 4; Additional files 2 and 3). More notably, 17,993 probes were found to be significantly different between orthotopic and ectopic models of Hep G2 (Fig. 5; Additional file 4).

Functional analysis of heat map of expression values using Gene Set Enrichment Analysis (GSEA) for explant 261004 showed upregulation of liver specific genes for orthotopically implanted tumors and downregulation for ectopically implanted tumors. As this was only observed in on sample, the difference could be due to reaction of murine enzymes to the xenograft. To prove this, further experiments involving purification of the samples to remove murine cells would be required.

Differences in gene expressions were also observed in orthotopically and subcutaneously inoculated explant 01-0207. Orthotopically-implanted tumors showed upregulation of genes for protein folding, response to biotic stimuli, enhanced drug-binding, response to stress and ubiquitin ligase complex formation. On the other hand, ectopically-implanted tumors showed up-regulation of genes for negative regulation of cellular processes.

\section{Discussion}

One of the biggest frustrations in drug development for $\mathrm{HCC}$ is the frequent failure to reproduce spectacular results from preclinical trials in a clinical setting. This has been attributed to the lack of clinically relevant animal models which can accurately predict the effects of a drug in the clinical setting. Currently, the predominant model consists of inoculating cell line suspension subcutaneously. Cell lines have a strong track record in cancer research due to their availability, rapid growth and ease of use. Ectopic models are also cheaper and less technically challenging than their orthotopic counterparts, thus allowing rapid screening of cytotoxic agents. However, it has been suggested that such models underestimate the influence of organ environment on subsequent tumorigenesis and metastasis. In this study, we aim to study the effects of the type of xenograft (cell line vs. patient tumor explant) and the site of xenograft inoculation (orthotopic vs. ectopic) on gene expression profiles and morphology of tumors.

A few conclusions can be drawn from our study. Firstly, gene expression of fresh tumors differed from the cell line and xenograft models, representing a drift in genotypic expression of the xenografts from originating tumors regardless of the site of implantation. It has been previously demonstrated by other authors that selection pressures in both cell-line and patient-derived xenograft models drive tumour growth which favours a more-aggressive sub-clone [41]. Neither model is a faithful representation of the heterogeneity seen in the original tumour which could explain the differences in gene expression seen in our study. Secondly, while cell line xenografts are distinct in gene expression from those of cells derived from patient tumor explants, there is no significant genotypic difference between xenografts that were orthotopically and ectopically implanted. That the cell line xenograft should differ from those derived from tumor explants is not a surprising conclusion having been previously demonstrated by several groups [42]. This lends credence to hypotheses that cell lines can become markedly unrepresentative of their parent tumors over time. Therefore, while cell lines are popular in research, our results show that they might be poor candidates as clinically relevant models in pharmacological studies. Cell lines are forced to adapt to a vastly different environment and this could lead to important characteristics of the original tumor to be selected against and eventually lost. Furthermore, there is also chance of cross-contamination of cultures and accumulation of mutations through passages [36, 42, 43]. However, we have only tested one cell line in this study and it is important to note that similar results may not hold true in other cell lines. More work still needs to be done to evaluate reproducibility in other established cell lines or discover and exclude those that hamper our efforts to bridge the preclinical-clinical divide.

In our study, the heat maps showed differences in orthotopically and ectopically implanted cell line models and 2 of the 3 patient explant models (261004 and 01-0207). Ectopically-implanted tumors appeared less metabolically active, responded less to biotic stimuli and external stresses, did not bind drugs and expressed higher levels of ubiquitin ligase, an enzyme involved in protein degradation. These could suggest biological bases for observed differences in preclinical and clinical drug response.

However, these differences in gene expression were not significant enough to cause a divergence in the dendrogram and PCA map. Tumors derived from the same originating explant clustered together, suggesting that the site of xenograft implantation has limited impact on gene expression. Other authors have previously shown that drug response was altered between orthotopic and ectopic murine models $[44,45]$. Taken together, the data suggests multifactorial influence on pharmacodynamics effects but organ microenvironment and tumor-host interactions could have more profound effects on drug 
response rather than gene expression. Indeed, studies have shown that invasive genotype and phenotype are affected by organ environment [44, 46]. Tumors transplanted subcutaneously were observed to be well-encapsulated, even when such feature was not apparent in the original tumor [47].

These results have implications on the choice of appropriate models. Although ectopic models have their limitations, our results showed that there is not much difference genetically between orthotopic and ectopic models. Unless organ-specific cellular targets are involved, ectopic models should still be considered for initial screening of drug target for their speed, ease of use and lower cost. Ectopic models would, however, be lacking in experiments studying the metastatic potential of HCC and the effect of autocrine and paracrine growth factors on the tumor. Furthermore, the liver is a complex organ with a vast and unique vasculature. The slow but extensive vasculature provides a favourable environment for tumors to establish, thrive and metas tasize [44]. In addition, proper functioning of liver in HCC patients is often complicated by cirrhosis, adding another layer of complexity which cannot be replicated in a subcutaneous model. An orthotopic model places the tumor in its native environment, allowing more evaluation of exposure levels of drug to tumor at an organ level, the rate of growth in the natural milieu and other tumor-host interactions. Therefore, the orthotopic model could be used to validate potential drug targets after initial screening.

\section{Conclusions}

In summary, our study showed that Hep G2 cells are genetically different from the other xenograft models. They also exhibited markedly different gene expression levels between orthotopic and ectopic sites and are probably a poor experimental choice for representing HCC. Tumor samples derived from patient explant clustered together according to their originating tumor. Heat maps showed different gene expression levels in explants 261004 and 01-0207 but these differences did not cause divergence of ectopic and orthotopic models on PCA mapping. This demonstrates that, despite the limitations, there could still be a role for ectopic models in drug screening, especially when its lower cost, rapidity and ease of use are considered. The lack of divergence in gene expression between orthotopic and ectopic models also suggests that tumor microenvironment and host-tumor interactions may have a greater impact on preclinical and clinical drug response disparity than gene expression. We suggest that ectopic models and orthotopic models can be complementary in their use; with ectopic models being used in initial target screening and orthotopic models used in the validation of potential drug targets or when more subtle organ-specific aspects need to be studied.

\section{Availability of supporting data}

The microarray data sets supporting the results of this article are available in the NCBI's Gene Expression Omnibus repository, http://www.ncbi.nlm.nih.gov/geo/ query/acc.cgi?acc=GSE72981. Other data sets supporting the results of this article are included within the article and its additional files published through LabArchives, DOI:10.6070/H4K07297 and hyperlink in https://mynotebook.labarchives.com/share/piercechow/ MjAuOHwxMTg1NjMvMTYvVHJlZU5vZGUvMjUwN Tk0MTM3MHw1Mi44.

\section{Additional files}

Additional file 1: Venn diagram illustrating the differences in gene expression in explant 261004, explant 01-0207 and Hep G2. (DOCX 365 kb)

Additional file 2: Expression profiling data of explant 261004. (XLS $110 \mathrm{~kb}$ )

Additional file 3: Expression profiling data of explant 01-0207. (XLS 38 kb)

Additional file 4: Expression profiling data of explant HEP G2. (XLS $2175 \mathrm{~kb}$ )

\section{Abbreviations}

HCC: Hepatocellular carcinoma; PCA: Principal component analyses; PDX: Patient-derived xenograft; HBV: Hepatitis B virus; HCV: Hepatitis C virus; SAPE: streptavidin-phycoerythrin conjugate; RMA: Robust Multichip Array; FDR: False discovery rate; GSEA: Gene set enrichment analysis.

\section{Competing interests}

The authors declare that they have no competing interests.

\section{Authors' contributions}

THT, KCK, HH and PKHC conceived, designed and coordinated the study. NGI participated in the design and coordination of the study. WW, WY and POBT performed the microarray PCA, provided the raw Affymetrix CEL files and participated in subsequent statistical analysis. ICS, LZ and HH performed all animal experiments in this study. TKHL provided histological expertise and assessed tissues for tumor content prior to extraction. THT, WW and NGI participated in the interpretation and analysis of results, drafted and finalized the manuscript. PKHC provided important data and vital subjects for research. All authors read and approved the final manuscript.

\section{Acknowledgements}

Microarray profiling was performed at the Duke-NUS Genome Biology Facility. This work is supported by National Medical Research Council (NMRC) [NMRC/ EDG/0021/2008] of Singapore.

\section{Author details}

${ }^{1}$ Cellular and Molecular Research, National Cancer Centre, Singapore 169610, Singapore. ${ }^{2}$ Department of Surgical Oncology, National Cancer Centre Singapore, Singapore 169610, Singapore. ${ }^{3}$ Department of General Surgery, Singapore General Hospital, 11 Hospital Drive, Singapore 169608, Singapore. ${ }^{4}$ Department of Histopathology, Singapore General Hospital, 11 Hospital Drive, Singapore 169608, Singapore. ${ }^{5}$ Sing Health Experimental Medicine Centre (SEMC), Blk 9, Level 3, Outram Road, Singapore 169608, Singapore. ${ }^{6}$ Division of Information Systems, School of Computer Engineering, Nanyang 
Technological University, Nanyang Avenue, Singapore 639798, Singapore. ${ }^{7}$ Laboratory of Molecular Endocrinology, Division of Molecular and Cellular Research, National Cancer Centre, 11 Hospital Drive, Singapore 169610, Singapore. ${ }^{8}$ Cancer and Stem Cell Biology Program, Duke-NUS Graduate Medical School, 8 College Road, Singapore 169857, Singapore. ${ }^{9}$ Program in Translational and Clinical Liver Research, National Cancer Centre Singapore, Singapore 169610, Singapore. ${ }^{10}$ Office of Clinical Sciences, Duke-NUS Graduate Medical School, 8 College Road, Singapore 169857, Singapore.

\section{Received: 19 January 2015 Accepted: 16 October 2015}

\section{Published online: 31 October 2015}

\section{References}

1. Maass T, Sfakianakis I, Staib F, Krupp M, Galle PR, Teufel A. Microarray-based gene expression analysis of hepatocellular carcinoma. Curr Genomics. 2010;11:261-8. doi:10.2174/138920210791233063.

2. Fujimoto A, Totoki Y, Abe T, Boroevich KA, Hosoda F, Nguyen HH, et al. Whole-genome sequencing of liver cancers identifies etiological influences on mutation patterns and recurrent mutations in chromatin regulators. Nat Genet. 2012;44:760-4. doi:http://www.nature.com/ng/journal/v44/n7/abs/ ng.2291.html\#supplementary-information.

3. Parkin DM. Global cancer statistics in the year 2000. Lancet Oncol. 2001;2:533-43. doi:10.1016/s1470-2045(01)00486-7.

4. Asia-Pacific Working Party on Prevention of Hepatocellular C. Prevention of hepatocellular carcinoma in the Asia-Pacific region: Consensus statements. J Gastroenterol Hepatol. 2010;25:657-63. doi:10.1111/j.1440-1746.2009.06167.x.

5. Venook AP, Papandreou C, Furuse J, Ladrón de Guevara L. The Incidence and Epidemiology of Hepatocellular Carcinoma: A Global and Regional Perspective. Oncologist. 2010;15:5-13. doi:10.1634/theoncologist.2010-S4-05.

6. Jemal A, Bray F, Center MM, Ferlay J, Ward E, Forman D. Global cancer statistics. CA Cancer J Clin. 2011;61:69-90. doi:10.3322/caac.20107.

7. lavarone M, Colombo M. HBV-related HCC, clinical issues and therapy. Dig Liver Dis. 2011:43:S32-9.

8. Benvegnù L, Fattovich G, Noventa F, Tremolada F, Chemello L, Cecchetto A, et al. Concurrent hepatitis $B$ and $C$ virus infection and risk of hepatocellular carcinoma in cirrhosis. A prospective study. Cancer. 1994;74:2442-8. doi:10.1002/1097-0142(19941101)74:9<2442::aid-cncr2820740909>3.0.co;2-\#.

9. Monto A, Wright TL. The epidemiology and prevention of hepatocellular carcinoma. Semin Oncol. 2001;28:441-9. doi:10.1016/s0093-7754(01)90137-x.

10. Fattovich G, Stroffolini T, Zagni I, Donato F. Hepatocellular carcinoma in cirrhosis: Incidence and risk factors. Gastroenterology. 2004;127:S35-50. doi:10.1053/j.gastro.2004.09.014.

11. Shariff MI, Cox IJ, Gomaa Al, Khan SA, Gedroyc W, Taylor-Robinson SD. Hepatocellular carcinoma: current trends in worldwide epidemiology, risk factors, diagnosis and therapeutics. Expert Rev Gastroenterol Hepatol. 2009:3:353-67. doi:10.1586/egh.09.35.

12. Llovet JM. Updated treatment approach to hepatocellular carcinoma. J Gastroenterol. 2005:40:225-35. doi:10.1007/s00535-005-1566-3.

13. Yuen M-F, Hou J-L, Chutaputti A. Hepatocellular carcinoma in the Asia pacific region. J Gastroenterol Hepatol. 2009;24:346-53. doi:10.1111/j.14401746.2009.05784.x.

14. Merican I, Guan R, Amarapuka D, Alexander M, Chutaputti A, Chien R, et al. Chronic hepatitis B virus infection in Asian countries. J Gastroenterol Hepatol. 2000;15:1356-61. doi:10.1046/j.1440-1746.2000. 0150121356.x

15. Chen C-J, Wang L-Y, Yu M-W. Epidemiology of hepatitis B virus infection in the Asia-Pacific region. J Gastroenterol Hepatol. 2000;15:E3-6. doi:10.1046/ j.1440-1746.2000.02124.x.

16. Di Bisceglie AM. Hepatitis B and hepatocellular carcinoma. Hepatology. 2009;49:S56-60. doi:10.1002/hep.22962.

17. de Villa V, Lo CM. Liver Transplantation for Hepatocellular Carcinoma in Asia. Oncologist. 2007;12:1321-31. doi:10.1634/theoncologist.12-11-1321.

18. Bosch FX, Ribes J, Díaz M, Cléries R. Primary liver cancer: Worldwide incidence and trends. Gastroenterology. 2004;127:S5-S16. doi:10.1053/j.gas tro.2004.09.011.

19. El-Serag HB. Hepatocellular Carcinoma: An Epidemiologic View. J Clin Gastroenterol. 2002;35:S72-8.

20. Bridges JFP, Joy S, Gallego G, Kudo M, Ye S-L, Han K-H, et al. Needs for hepatocellular carcinoma control policy in the Asia-Pacific region. Asian Pac J Cancer Prev. 2011;12:2585-91.
21. Cabibbo G, Enea M, Attanasio M, Bruix J, Craxi A, Camma C. A metaanalysis of survival rates of untreated patients in randomized clinical trials of hepatocellular carcinoma. Hepatology. 2010;51:1274-83. doi:10.1002/hep.23485

22. Cucchetti A, Piscaglia F, Cescon M, Ercolani G, Terzi E, Bolondi L, et al. Conditional survival after hepatic resection for hepatocellular carcinoma in cirrhotic patients. Clin Cancer Res. 2012. doi:10.1158/1078-0432.ccr-11-2663.

23. Ho W-H, Lee K-T, Chen H-Y, Ho T-W, Chiu H-C. Disease-Free Survival after Hepatic Resection in Hepatocellular Carcinoma Patients: A Prediction Approach Using Artificial Neural Network. PLoS One. 2012;7:e29179. doi:10.1371/journal.pone.0029179.

24. Figueras J, Jaurrieta E, Valls C, Benasco C, Rafecas A, Xiol X, et al. Survival after liver transplantation in cirrhotic patients with and without hepatocellular carcinoma: A comparative study. Hepatology. 1997;25:1485-9. doi:10.1002/hep.510250629.

25. Lee PH, Lin WJ, Tsang YM, Hu RH, Sheu JC, Lai MY, et al. Clinical management of recurrent hepatocellular carcinoma. Ann Surg. 1995;222:670-6.

26. Miyazaki M, Kimura F, Shimizu H, Yoshidome H, Ohtsuka M, Kato A, et al. Surgical Treatment for Liver Cancer. Dig Surg. 2007;24:120-5.

27. Takayama T. Surgical Treatment for Hepatocellular Carcinoma. Jpn J Clin Oncol. 2011;41:447-54. doi:10.1093/jjco/hyr016.

28. Ryder SD. Guidelines for the diagnosis and treatment of hepatocellular carcinoma (HCC) in adults. Gut. 2003;52:iii1-8. doi:10.1136/ gut.52.suppl_3.iii1.

29. Deshpande R, Reilly D, Sherlock D. Improving Outcomes with Surgical Resection and Other Ablative Therapies in HCC. J Hepatol. 2011;2011. doi:10.4061/2011/686074.

30. Feng YX, Wang T, Deng YZ, Yang P, Li JJ, Guan DX, et al. Sorafenib suppresses postsurgical recurrence and metastasis of hepatocellular carcinoma in an orthotopic mouse model. Hepatology. 2011;53:483-92. doi:10.1002/hep.24075

31. Llovet JM, Ricci S, Mazzaferro V, Hilgard P, Gane E, Blanc J-F, et al. Sorafenib in Advanced Hepatocellular Carcinoma. N Engl J Med. 2008;359:378-90. doi:10.1056/NEJMoa0708857.

32. Johnson Jl, Decker S, Zaharevitz D, Rubinstein LV, Venditti JM, Schepartz S, et al. Relationships between drug activity in $\mathrm{NCl}$ preclinical in vitro and in vivo models and early clinical trials. Br J Cancer. 2001;84:1424-31. doi:10.1054/bjoc.2001.1796

33. Borrell B. How accurate are cancer cell lines? Nature. 2010;463:858. doi:10.1038/463858a.

34. Leenders MW, Nijkamp MW, Borel Rinkes $\mathbf{I H}$. Mouse models in liver cancer research: a review of current literature. World J Gastroenterol. 2008;14:6915-23.

35. Sano D, Myers JN. Xenograft models of head and neck cancers. Head Neck Oncol. 2009;1:32. doi:10.1186/1758-3284-1-32.

36. Correia AL, Bissell MJ. The tumor microenvironment is a dominant force in multidrug resistance. Drug Resist Updat. 2012;15:39-49. doi:10.1016/j.drup. 2012.01.006

37. Heijstek MW, Kranenburg O, Borel Rinkes $1 \mathrm{H}$. Mouse models of colorectal cancer and liver metastases. Dig Surg. 2005;22:16-25. doi:10.1159/000085342.

38. Glazer ES, Stone EM, Zhu C, Massey KL, Hamir AN, Curley SA. Bioengineered human arginase I with enhanced activity and stability controls hepatocellular and pancreatic carcinoma xenografts. Transl Oncol. 2011;4:138-46.

39. Irizarry RA, Hobbs B, Collin F, Beazer-Barclay YD, Antonellis KJ, Scherf U, et al. Exploration, normalization, and summaries of high density oligonucleotide array probe level data. Biostatistics. 2003;4:249-64. doi:10.1093/biostatistics/4.2.249.

40. Irizarry RA, Bolstad BM, Collin F, Cope LM, Hobbs B, Speed TP. Summaries of Affymetrix GeneChip probe level data. Nucleic Acids Res. 2003;31:e15.

41. Aparicio $S$, Hidalgo M, Kung AL. Examining the utility of patient-derived xenograft mouse models. Nat Rev Cancer. 2015;15:311-6. doi:10.1038/nrc3944.

42. Kato S, Espinoza N, Lange S, Villalon M, Cuello M, Owen Gl. Characterization and phenotypic variation with passage number of cultured human endometrial adenocarcinoma cells. Tissue Cell. 2008;40:95-102. doi:10.1016/j.tice.2007.09.007.

43. Cree IA, Glaysher S, Harvey AL. Efficacy of anti-cancer agents in cell lines versus human primary tumour tissue. Curr Opin Pharmacol. 2010;10:375-9. doi:10.1016/j.coph.2010.05.001.

44. Wilmanns C, Fan D, O'Brian CA, Bucana CD, Fidler IJ. Orthotopic and ectopic organ environments differentially influence the sensitivity of murine colon carcinoma cells to doxorubicin and 5-fluorouracil. Int J Cancer. 1992;52:98-104. 
45. Kuo TH, Kubota T, Watanabe M, Furukawa T, Kase S, Tanino H, et al. Sitespecific chemosensitivity of human small-cell lung carcinoma growing orthotopically compared to subcutaneously in SCID mice: the importance of orthotopic models to obtain relevant drug evaluation data. Anticancer Res. 1993;13:627-30

46. Liotta LA, Kohn EC. The microenvironment of the tumour-host interface. Nature. 2001;411:375-9. doi:10.1038/35077241.

47. Kubota T. Metastatic models of human cancer xenografted in the nude mouse: the importance of orthotopic transplantation. J Cell Biochem. 1994;56:4-8. doi:10.1002/jcb.240560103.

Submit your next manuscript to BioMed Central and take full advantage of:

- Convenient online submission

- Thorough peer review

- No space constraints or color figure charges

- Immediate publication on acceptance

- Inclusion in PubMed, CAS, Scopus and Google Scholar

- Research which is freely available for redistribution 